\title{
Zero Sum, the Niche, and Metacommunities: Long-Term Dynamics of Community Assembly
}

\author{
S. K. Morgan Ernest, ${ }^{1, *}$ James H. Brown, ${ }^{2, \dagger}$ Katherine M. Thibault, ${ }^{3, \uparrow}$ Ethan P. White, ${ }^{1,4, \S}$ and Jacob R. Goheen ${ }^{5, \|}$
}

1. Department of Biology and the Ecology Center, Utah State University, Logan, Utah 84322;

2. Department of Biology, University of New Mexico,

Albuquerque, New Mexico 87131;

3. Department of Biology, Furman University, Greenville, South Carolina 29613;

4. Department of Ecology and Evolutionary Biology, University of Arizona, Tucson, Arizona 85721;

5. Department of Zoology, University of British Columbia,

Vancouver, British Columbia V6T 1Z4, Canada

Submitted August 28, 2007; Accepted August 7, 2008;

Electronically published October 23, 2008

AвSTRACT: Recent models of community assembly, structure, and dynamics have incorporated, to varying degrees, three mechanistic processes: resource limitation and interspecific competition, niche requirements of species, and exchanges between a local community and a regional species pool. Synthesizing 30 years of data from an intensively studied desert rodent community, we show that all of these processes, separately and in combination, have influenced the structural organization of this community and affected its dynamical response to both natural environmental changes and experimental perturbations. In addition, our analyses suggest that zero-sum constraints, niche differences, and metacommunity processes are inextricably linked in the ways that they affect the structure and dynamics of this system. Explicit consideration of the interaction of these processes should yield a deeper understanding of the assembly and dynamics of other ecological communities. This synthesis highlights the role that long-term data, especially when coupled with experimental manipulations, can play in assessing the fundamental processes that govern the structure and function of ecological communities.

\footnotetext{
* E-mail: morgane@biology.usu.edu.

† E-mail: jhbrown@unm.edu.

₹ E-mail: katherine.thibault@furman.edu.

${ }^{\S}$ E-mail: epwhite@biology.usu.edu.

" E-mail: goheen@zoology.ubc.ca.

Am. Nat. 2008. Vol. 172, pp. E257-E269. (c) 2008 by The University of Chicago. 0003-0147/2008/17206-42827\$15.00. All rights reserved. DOI: $10.1086 / 592402$
}

Keywords: community dynamics, neutral theory, interspecific competition, resource limitation, species sorting, Chihuahuan Desert.

Despite recent claims that there are no laws in community ecology (Lawton 1999; Simberloff 2004), the past decade has witnessed a resurgence of interest in the general processes that determine community structure and dynamics. One result is the development of several mechanistic models of community assembly (e.g., Hubbell 2001; Chase and Leibold 2003; Leibold et al. 2004; Tilman 2004). Some of these models are based on such traditional concepts as competition and niche theory (e.g., Chase and Leibold 2003; Tilman 2004), whereas others focus on the roles of stochastic demographic and dispersal processes (e.g., Hubbell 2001). Regardless of the approach, this renewed search for generality has revitalized debate and motivated research into the mechanistic underpinnings of community ecology (e.g., Chave et al. 2002; Chase and Leibold 2003; McGill 2003b; Adler 2004; Snyder and Chesson 2004; Tilman 2004; Hubbell 2005, 2006; Wootton 2005; Holyoak and Loreau 2006; Leibold and McPeek 2006).

Recent models of assembly differ in the extent to which they emphasize demographic and niche differences among species, dispersal-limitation, and stochastic processes (e.g., Hubbell 2001; Mouquet and Loreau 2003; Kneitel and Chase 2004; Leibold et al. 2004; Snyder and Chesson 2004; Tilman 2004; Gravel et al. 2006; Adler et al. 2007). Despite differences in emphasis, however, most of these models incorporate some subset of three common processes: a resource constraint, niches that characterize overlap among species in resource utilization, and exchanges of species and individuals between a local community and a regional species pool. The models differ, however, in how they parameterize these processes and in the extent to which each is emphasized. For example, Hubbell's (2001) neutral theory proposes that colonization from a regional species pool and stochastic birth/death and speciation events are the primary determinants of the relative abundance, composition, and diversity of species in local communities (see also Caswell 1976; Bell 2001). This theory is based on the controversial assumption that species have 
identical per capita demographic rates (technically, replacement probabilities) and that niche differences, although they certainly exist, are effectively neutral and therefore do not play an important role in determining the local abundances and distributions of species (Hubbell 2001, 2005). Alternative models emphasize the roles of resource limitation and niche differences among species while still incorporating stochastic elements of dispersal and demography (e.g., Tilman 2004; Gravel et al. 2006). Consequently, these differences have led to extensive debate surrounding the fundamental processes involved in community assembly.

Such debates remain unresolved, in part because multiple models predict the same community patterns (Chave et al. 2002, 2006; McGill 2003a; Mouquet and Loreau 2003; Volkov et al. 2005, 2006; Nekola and Brown 2007) and in part because the alternative processes are not really mutually exclusive. The traditional approach to evaluating these models, and thus the processes they incorporate, involves choosing one or a few observable measures of community structure and comparing them to the predictions of one or more models (e.g., McGill 2003b; Adler 2004; Volkov et al. 2005). An alternative approach is to take an in-depth, synthetic look at well-studied communities in an attempt to infer underlying processes. This approach may be particularly informative if the study combines long-term observational data with experimental manipulations. To our knowledge, this approach has yet to be taken in the context of recent community models.

Here we take this alternative approach, synthesizing 30 years of research on a desert rodent community, in an attempt to improve our understanding of how zero-sum dynamics, niches, and dispersal limitation/metacommunity dynamics combine to structure local communities. We recognize that research at a single site cannot definitively answer these questions. However, community assembly depends on dynamic processes (i.e., fluctuations in abundance, colonization, and extinction), and longterm experiments can provide unique insights into the processes structuring communities. These insights can then be used to inform the next generation of community models. Our long-term data can advance our understanding of community assembly by revealing how these processes act and interact to determine the abundance, distribution, and diversity of species.

\section{Background on Portal}

In 1977, J. H. Brown and his associates initiated an experimental manipulation of ants, plants, and rodents in the Chihuahuan Desert near the town of Portal, Arizona. The 20-ha site (elevation $1,330 \mathrm{~m}$ ) is fenced to exclude cattle and is located at the transition between Chihuahuan
Desert scrub and higher-elevation arid grasslands. The Portal Project consists of 24 fenced plots, each 0.25 ha in size $(50 \mathrm{~m} \times 50 \mathrm{~m})$. Each plot is associated with a permanent sampling grid, where sampling points are marked by a $7 \times 7$ grid of rebar. At the outset of the project, treatments were allocated randomly to plots. These treatments excluded different groups of rodents, with some plots reserved as controls to which all rodents had access. Gates in the fences exclude or permit access by rodents to the plots, with smaller gates excluding the larger rodent species, namely, the kangaroo rats (Dipodomys spp.).

Originally, the study was designed to investigate interactions among granivores (rodents and ants) and impacts of these animals on annual plants. Over the 30 years, research has addressed how the structure and dynamics of this ecosystem are affected by interspecific competition (Munger and Brown 1981; Bowers and Brown 1982; Brown and Munger 1985; Heske et al. 1994; Brown et al. 2000), indirect interactions (Davidson et al. 1984; Brown and Heske 1990; Thompson et al. 1991; Smith et al. 1997), colonization-extinction dynamics (Valone and Brown 1995; Valone et al. 1995; Brown et al. 2001; Ernest and Brown 2001a; Goheen et al. 2005), extreme climatic events (Thibault and Brown 2008), and resource limitation (Brown and Munger 1985; Bowers et al. 1987; Ernest et al. 2000; Ernest and Brown 2001a, 2001b; White et al. 2004). Importantly, the long-term manipulations and monitoring have been conducted against a background of major environmental change. Woody plants increased and grasses decreased dramatically from the mid-1970s to the mid-1990s, likely because of long-term changes in precipitation (Brown et al. 1997) that favored shrubs (Neilson 1986). This conversion of habitat and coincident changes in species composition provide a unique opportunity to document mechanisms of community assembly in response to a major environmental change.

Because the rodent community has been the most intensively and consistently studied community at the site, we focus this synthesis on that group. Desert rodents are a particularly informative group because of the diversity of coexisting granivorous rodent species at Portal that overlap in requirements for the same limiting resourceseeds - and because many aspects of the ecology of desert rodents are well understood (e.g., Kotler 1984; Kotler and Brown 1988; Genoways and Brown 1993; Brown et al. 1994). Here we focus primarily on the natural dynamics of the rodent community occurring on the control plots, with specific discussion of results from the kangaroo ratremoval plots when appropriate. This unique combination of a well-understood group of study organisms, long-term monitoring, experimental manipulation, and background environmental change has yielded insights into the im- 
portance of and interactions among resource competition, niche relationships, and metacommunity dynamics.

\section{Resource Limitation, Competition, and Zero-Sum Dynamics}

The assumption of resource limitation provides a foundation for much of ecological and evolutionary theory (e.g., MacArthur and Levins 1967; Van Valen 1973, 1976; Schoener 1974; Levinton 1979; Tilman 1982). In community ecology, resource limitation can manifest as a zerosum dynamic (also sometimes referred to as communitylevel carrying capacity; Brown 1981; Wright 1983; Ernest and Brown 2001b; Hubbell 2001; del Monte-Luna et al. 2004). Recently, the role of zero-sum dynamics has been scrutinized, largely because it is stated by Hubbell (2001) to be a central assumption of neutral theory. While it has recently been shown that the zero-sum assumption does not influence the distribution of abundances predicted by the neutral theory (Etienne et al. 2007), it can influence other behaviors of this and other models that incorporate it as an assumption. Specifically, in the neutral theory, some resource is assumed to be limiting at the level of the entire community, thereby setting a fixed limit on the total number of individuals. Inherent to this idea is the prediction that species should exhibit density compensation: increases in one species should be compensated for by decreases in other species, assuming that the limiting resource is not changing. Recent work has challenged the idea that competition for limiting resources drives abundance dynamics by showing that, in many communities, species' populations fluctuate in synchrony, suggesting that the long-term dynamics of abundance reflect similar responses to environmental changes across species (Houlahan et al. 2007). These seemingly conflicting viewpoints have important implications for how community dynamics should be modeled, analyzed, and interpreted.

Our long-term site at Portal provides a critical lesson for understanding how resource limitation operates in communities, namely, that abundance is not directly related to the rate of resource consumption and therefore allows questions about zero-sum dynamics to be addressed only indirectly and imprecisely. Because consumption rates are determined by metabolic rate, which is related to body size (e.g., Kleiber 1932; Peters 1983; Calder 1984; West et al. 1997), abundance will not directly reflect resource use in communities where species differ in size (i.e., in almost all natural communities). The same rate of resource supply can support either fewer large organisms or more smaller organisms because large organisms have higher per capita rates of consumption than small ones (Damuth 1981; Peters 1983; Calder 1984; Ernest and Brown 2001b; Brown et al. 2004; White et al. 2007). Integrating metabolic theory
(Brown et al. 2004) and zero-sum dynamics allows questions concerning the role of resource limitation in communities to be recast and evaluated directly in the context of resource supply and utilization (Ernest and Brown 2001b; White et al. 2004). This is accomplished by analyzing communities using an estimate of community-level energy (i.e., resource) use (hereafter referred to as "aggregrate energy use") based on individual metabolic rates calculated from well-known allometries (e.g., Kleiber 1932). In North American deserts, rodents are the dominant consumer of seeds, and multiple lines of evidence demonstrate that seed availability limits their abundance (Brown et al. 1979; Brown and Munger 1985; Kelt et al. 2004). Therefore, a reasonable assumption in this system is that resource use approximates resource supply.

Under a zero-sum steady state of rates of resource supply and consumption, a metabolic framework makes three predictions: (1) if species composition changes through time, the variability in species composition should be greater than the variability in the aggregate energy use for a community (Ernest and Brown 2001b); (2) significant negative covariances in energy use should exist among species (Ernest and Brown 2001b); and (3) if the average body size of an individual changes in a community, then the number of individuals should also change, with the change in abundance being proportional to the change in the inverse of average metabolic rate (White et al. 2004). All of these predictions are supported by the long-term data on seed-eating rodents at Portal. Aggregate energy use did not change directionally through time despite high variability in species composition (fig. 1, bottom; Ernest and Brown 2001b; White et al. 2004). Covariance ratio tests (e.g., Frost et al. 1995) demonstrate negative covariances in energy use among species, indicating greater stability than expected from the fluctuations of individual species $(P=.039)$. In contrast, abundance exhibited positive covariances suggestive of synchronized dynamics $(P=.032)$. If resources are highly variable through time, abundance could exhibit positive covariances even if a community is operating under resource limitation, but the fact that abundance and energy use show different covariances (positive and negative, respectively) suggests that the positive covariance in abundance is not due solely to variability in resource supply. The different results obtained by examining abundance and energy use highlight the problems with conducting these analyses on currencies decoupled from resources.

Part of the reason for the different responses of energy use and abundance is that we have serendipitously witnessed a size shift at the site, thus allowing us to evaluate the predicted trade-off between size and abundance. Since 1977, changes in species composition resulted in the average body size of an individual decreasing from approx- 

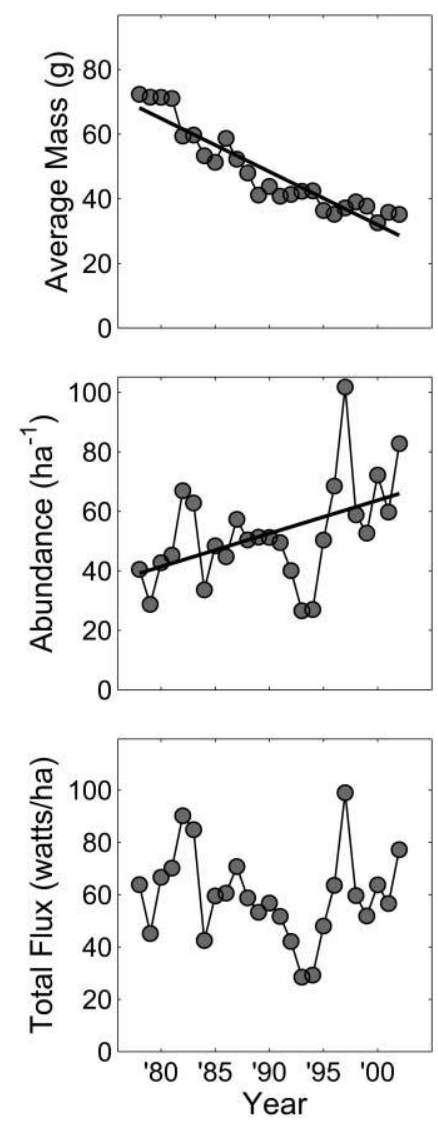

Figure 1: Temporal trends (1978-2002) for the granivorous small-mammal community at Portal, Arizona. Observed trends in average individual mass (top, g), total abundance (middle, individuals/ha), and summed metabolic rate, an index of community-level energy use (bottom, W/ha). Regression lines represent significant linear relationships between year and the variable of interest.

imately 80 to $30 \mathrm{~g}$. Concomitantly, total abundance of rodents almost doubled, and total biomass decreased (fig. 1; White et al. 2004). As predicted by the framework, the increase in abundance was inversely proportional to changes in average metabolic rate (see White et al. 2004 for details). Taken together, the combination of the stability of aggregate energy use, negative covariances in energy use by populations, and the trade-off between body size and abundance demonstrates that the desert rodent community at Portal is conforming to predictions from an energy-based zero-sum dynamic.

While the results from Portal suggest that zero-sum dynamics could be more prevalent than recently suggested (Houlahan et al. 2007), observations at the site also suggest that a single timescale is insufficient to characterize a community as exhibiting competitive or synchronous dynamics. Different processes operating at different timescales can drive the system to be more competitive or more synchronous (e.g., Vasseur et al. 2005). Portal has experienced both short-term fluctuations in resource supply and long-term changes in habitat. On shorter timescales of months to a few years, resources fluctuate in response to precipitation, plant and insect production, and other factors (e.g., note the decline in energy use in the mid1990 s that resulted from an extended drought in the region; fig. 1, bottom). Consequently, even strongly competing rodent species typically show positively correlated fluctuations in abundance: a good season for one species is a good season for all (Brown and Heske 1990; Valone and Brown 1996). Similar population responses across multiple species to resource pulses have been documented in a number of systems (e.g., Ostfeld and Keesing 2000; Meserve et al. 2001; Ruhl and Smith 2004; Vasseur et al. 2005; Holmgren et al. 2006; Valone and Barber 2008). On longer timescales, major compositional shifts have occurred at Portal in response to a shift in habitat from desert grassland to shrubland (fig. 2; Brown et al. 1997; Thibault et al. 2004). Regular increases in shrublandaffiliated species and decreases in grassland-affiliated spe-

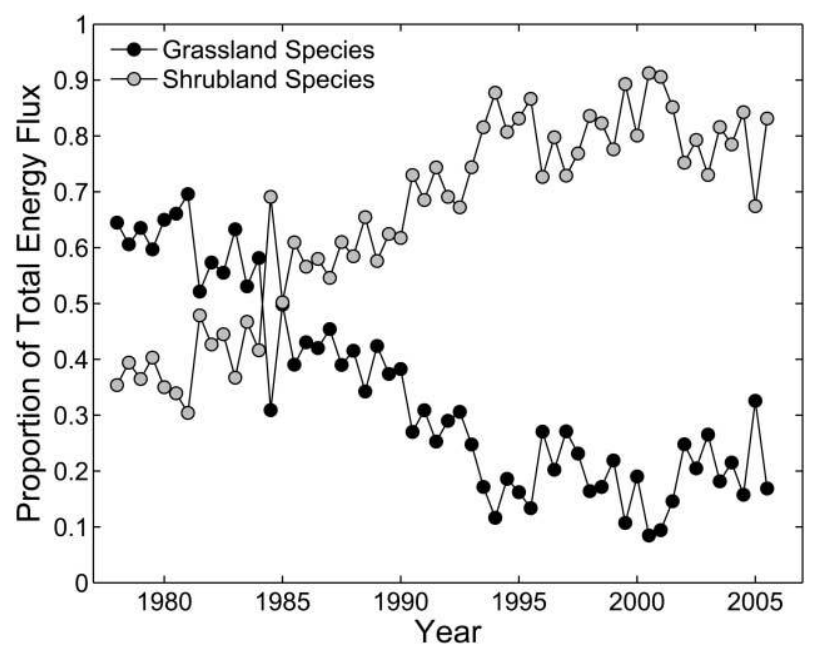

Figure 2: Temporal trends (1978-2005) in the proportion of total energy flux of the Portal granivorous rodent community used by grasslandaffiliated species (black circles) and shrubland-affiliated species (gray circles). We present both lines for illustrative purposes, even though the values for grassland species are equal to 1 minus the values for shrubland species. The temporal trend for the increase in shrubland species is statistically significant $\left(r^{2}=0.74 ; P<.001\right)$. Habitat affinities are based on Hoffmeister (1986) as follows. Grassland species: Baiomys taylori, Chaetodipus hispidus, Dipodomys ordii, Dipodomys spectabilis, Perognathus flavus, Peromyscus leucopus, Peromyscus maniculatus, Reithrodontomys fulvescens, Reithrodontomys megalotis, Reithrodontomys montanus, Sigmodon fulviventer, Sigmodon hispidus, and Sigmodon ochrognathus. Shrubland species: Chaetodipus baileyi, Chaetodipus intermedius, Chaetodipus penicillatus, Dipodomys merriami, and Peromyscus eremicus. 
cies (see "The Niche" for details) result in the observed negative covariances in energy use (Ernest and Brown $2001 \mathrm{~b}$ ). Some recent studies suggest that the observation of synchronous responses at some timescales and compensatory responses at others may be a common feature of ecological communities (Brady and Slade 2004; Keitt and Fischer 2006; Vasseur and Gaedke 2007). While these studies were conducted on abundance or biomass and not more direct estimates of resource use, it seems likely that timescale effects will also occur with more appropriate currencies.

Results from our long-term study call into question the recent suggestion (Houlahan et al. 2007) that competitively driven compensatory dynamics are uncommon in ecological communities. First, the analyses by Houlahan et al. (2007) specifically used negative covariation in numbers of individuals (i.e., abundance) to assess compensation (Root 1973). Had we used abundance to analyze the longterm dynamics of this desert rodent community, we would have concluded that the community dynamics were dominated by positive covariation among species when in fact they were dominated by competitive (i.e., negative) compensatory dynamics. Perhaps more importantly, recent studies suggest that the questions of interest should not be whether a site exhibits compensatory or synchronous dynamics but rather at what timescales different processes dominate the observed dynamics and how processes at different timescales affect stability of community properties (see Vasseur and Gaedke 2007).

\section{The Niche}

While stochastic processes and neutral models have been employed in community ecology for decades (e.g., MacArthur 1957; MacArthur and Wilson 1967; Caswell 1976; Hubbell 1979; Hughes 1984; Bell 2000), the theory recently proposed by Hubbell (2001) has spurred spirited debate about the relevance of the niche concept for understanding community assembly, structure, and dynamics (e.g., Bell 2001; Enquist et al. 2002; McGill 2003b; Adler 2004; Wootton 2005; Hubbell 2006). Because Hubbell's (2001) model, which assumes functional equivalence among species, appears to predict community structure as well as or better than many niche-based models (Hubbell 2001; Volkov et al. 2003), it has challenged ecologists to critically reexamine whether niche-based hypotheses are necessary or sufficient to account for community-level phenomena.

A critical contemporary issue in ecology concerns the response of communities to environmental change. Neutral theory predicts that species are demographically neutral-that is, they have equal per capita probabilities of birth and death and of colonization and extinction-not only under static environmental conditions but also in response to changes in climate, habitat, and other factors (Hubbell 2001). Niche-based models, by contrast, predict that in a constant environment, the species abundance distribution will reflect niche differences among species. However, once this distribution has been achieved, the species will have equal fitness, so that fluctuations will be small and may be difficult to distinguish from random changes (Chave 2004). More importantly, however, nichebased models predict that abundances will change rapidly and predictably-and potentially much faster than predicted by demographic neutrality - in response to substantial environmental change. When the environment changes, the niche differences among species will cause changes in the relative fitnesses of individuals and in the demography of populations, resulting in deterministic changes in abundance and species composition (e.g., Chesson 2000). It is not surprising, therefore, that niche-based and neutral models may appear to perform nearly equally well when evaluated against relatively short-term data. Long-term data, however, should allow differentiation between niche-based and neutral models. If the environment remains constant over long periods, then community composition should be more stable than expected from neutrality (Clark and McLachlan 2003; McGill et al. 2005). Alternatively, if the environment changes rapidly, as is predicted to occur in practically all global change scenarios, changes in abundance and composition of species should be much more rapid than predicted by neutrality and should proceed in directions predicted on the basis of the niche requirements of the species.

Long-term data from Portal provide strong support for niche-based community dynamics. The strongest evidence comes from the long-term trends in the abundances of individual species in response to climate and vegetation change. The threefold increase in shrub density since 1977 resulted in a dramatic transition in habitat from open desert grassland to a mixture of grasses and shrubs (Brown et al. 1997), as well as dramatic changes in the composition of the rodent community. Several species abundant at the beginning of the study became either extinct or very rare, while other species colonized and/or increased dramatically in abundance (Valone et al. 1995; Brown et al. 1997; Thibault et al. 2004). Although these rapid changes in species composition suggest an important role for niches, the neutral model also predicts that composition should drift, albeit relatively slowly, over time. Importantly, however, many of these observed changes are predictable on the basis of known niche characteristics of the species. For example, as indexed by energy use, grassland-affiliated species declined significantly over time, whereas shrublandaffiliated species increased dramatically (fig. 2). The fact that the responses of species reflected their habitat affinities indicates that the marked changes in species abundances 
and community composition were due primarily to niche differences.

In addition to the directional changes in species abundance and community composition, the individual size distribution (sensu White et al. 2007) also changed directionally (see "Resource Limitation, Competition, and Zero-Sum Dynamics"; White et al. 2004). This change may have been due in part to idiosyncrasies in the relationship between habitat affiliation and species body size. For example, the kangaroo rat that became locally extinct, $D i$ podomys spectabilis, was the largest species and a grassland specialist. However, grassland-affiliated species also included the smallest species that occurred at the site (Reithrodontomys megalotis, $11 \mathrm{~g}$, and Baiomys taylori, $8 \mathrm{~g}$ ), and these two species also declined. In addition, the decrease in size is present even when D. spectabilis is excluded from the analysis (White et al. 2004), suggesting that the change in size structure is not driven solely by one species but rather may be due to niche characteristics related to size. Studies on desert rodents show that many important foraging characteristics are related to size (e.g., Price 1983, 1993; Brown 1989; Morgan and Price 1992; Reichman and Price 1993; Price and Mittler 2003, 2006). This, together with empirical evidence of body size partitioning of resources (Bowers and Brown 1982; Ernest and Brown 2001a; Ernest 2005), strongly suggests that the shift in the size distribution reflects a temporal shift in the niches available at Portal.

Niche characteristics also play an important role in the compensatory dynamics that maintain the zero sum. Instead of random replacement of individuals, as predicted by neutrality (Hubbell 2001), the dynamics at Portal showed that species replacements and compensation were based on niche characteristics. One example is the replacement of grassland species with shrubland species discussed above. This compensation was probably governed by well-documented foraging trade-offs whereby species trade off foraging efficiencies along one or more axes (see Kotler and Brown 1988 for review). In our system, two nonexclusive trade-offs could be operating. First, species may trade foraging efficiency (i.e., seeds harvested per unit time) for reduced travel costs (Kotler and Brown 1988; Richards et al. 2000; Chase et al. 2001). Previous research has demonstrated that for bipedal and/or large-bodied rodents, reduced travel costs result in the quicker discovery of, and departure from, resource patches (Brown 1989; Brown et al. 1994). Because seed patches tend to be more widely spaced in open desert grasslands than in shrublands, altered resource distributions could select for smaller-bodied species that can forage more efficiently in the shrubby habitat (Reichman and Oberstein 1977; Price 1978). Second, species may experience reduced risk of predation, at the expense of foraging efficiency in bushy mi- crohabitats, relative to that in open microhabitats (Kotler et al. 1994). Dipodomys spp. exhibit traits such as inflated auditory bullae and bipedal locomotion to detect and evade predators while foraging in open microhabitats, and they forage less efficiently in bushy microhabitats than smaller-bodied, quadrupedal Chaetodipus species (Kotler 1984; Brown et al. 1988). Such trade-offs may have enabled the rodent community to respond quickly to environmental changes, by enabling a diverse group of species to coexist and reorganize in the face of long-term habitat conversion.

The importance of niche characteristics in maintaining a zero-sum game is best exemplified by the long-term dynamics on kangaroo rat-removal plots. When kangaroo rats were experimentally removed, the energy used by the remaining small granivores initially increased but not nearly enough to compensate for the missing kangaroo rats. In fact, the small granivores utilized, on average, only $14 \%$ of the energy made available by the exclusion of kangaroo rats. Not until a new species, Chaetodipus baileyi, colonized the site in 1995 did energy use on kangaroo ratremoval plots increase to near control levels. By 1999, energy use by this one species alone approached $80 \%$ of the energy used by the kangaroo rats on the control plots (fig. 3; Ernest and Brown 2001a). This level of energetic compensation by a single species suggests that the niches of kangaroo rats and C. baileyi overlap substantially. It is almost certainly not coincidental that $C$. baileyi is much more similar in size to kangaroo rats than to the small granivores that were unable to compensate for kangaroo

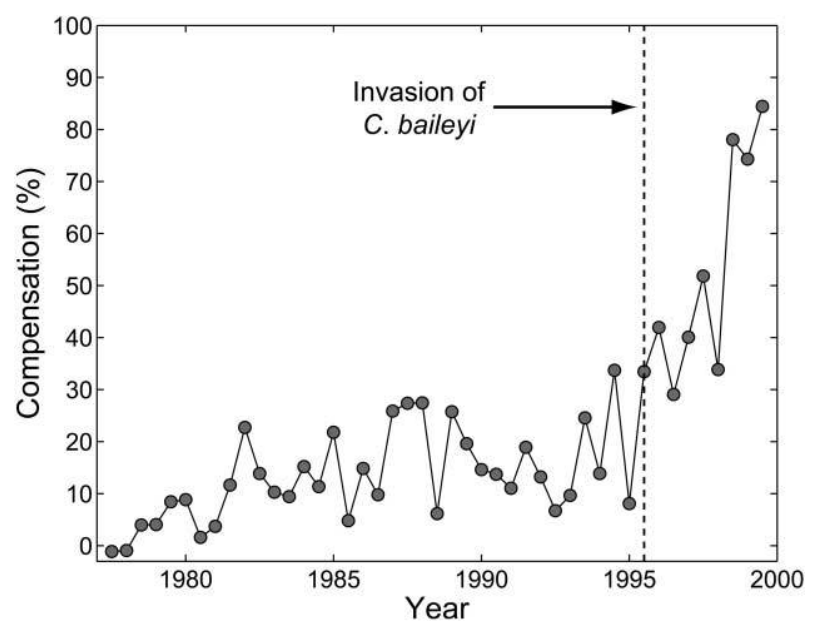

Figure 3: Percent of the energy used by the non-Dipodomys granivores on kangaroo rat-removal plots relative to the total energy used by kangaroo rats (Dipodomys spp.) on control plots (modified from Ernest and Brown 2001a). The dashed line indicates the time of colonization of the site by Chaetodipus baileyi. 
rat removal (Ernest and Brown 2001a). So, a kangaroo rat niche was made available by kangaroo rat removal, and it remained underexploited until a species with the appropriate characteristics colonized, increased, used most of the previously unused resources, and virtually restored the zero sum. Niche differences were therefore critical in maintaining zero-sum dynamics in response to each of two distinct perturbations: experimental species removal and background habitat change. In addition, results from the kangaroo rat-removal plots clearly show that particular species have profound effects on other aspects of community structure. Experimental removal of kangaroo rats resulted in higher abundances, species richness, and colonization rates and lower extinction rates for the smallgranivore group (Valone and Brown 1995). So, contrary to predictions of the neutral model (Hubbell 2001), where colonization and extinction rates are stochastic and unrelated to niche characteristics or competitive interactions, at Portal dispersal and establishment depended strongly on which species were present in the community.

Finally, unlike some niche-based models that focus primarily on niche differences in resource utilization (e.g., MacArthur and Levins 1967; Tilman 1982), the results at Portal suggest that the non-food-related components of the niche (e.g., predation risk related to microhabitat structure) are critical for understanding long-term dynamics of species composition. Other recent models (e.g., Chesson 2000; Chase and Leibold 2003) have explored how niche differences in both resource use and response to climate can promote coexistence. Our results suggest that these approaches may be particularly valuable for understanding the long-term dynamics of communities where abiotic conditions fluctuate or change directionally through time.

While niche differences are clearly important for the Portal rodent community, this does not mean that demographic stochasticity is not important. Demographic stochasticity has been shown to influence population and community dynamics in a number of different systems (Underwood and Fairweather 1989; Hubbell 2001 and references therein) and presumably also plays a role at Portal. However, our results do indicate that a full understanding of the dynamics of at least some types of ecological systems requires the incorporation of niche differences into community models.

\section{Metacommunity Dynamics}

MacArthur and Wilson's (1967) Theory of Island Biogeography challenged ecologists to think about the spatial context in which a local community is embedded. Forty years later, we are still working to answer the basic questions: to what extent do the composition and dynamics of a local community reflect the diversity and composition of the regional species pool, and to what extent are they determined by local processes (e.g., Karlson and Cornell 2002; Holyoak et al. 2007; Stevens et al. 2007; Werner et al. 2007)? While many contemporary models incorporate dispersal from a species pool or metacommunity, they differ in their predictions about the influence of local and regional processes (e.g., Hubbell 2001; Mouquet and Loreau 2003; Snyder and Chesson 2004; Gravel et al. 2006). Four general classes of metacommunity models have been proposed to characterize connections between a local community and the communities linked to that site through dispersal (Leibold et al. 2004): neutral, patch, mass effect, and species-sorting models. These models differ mainly in the extent to which the local community, because of some combination of species interactions and abiotic conditions, is open to exchange with the metacommunity.

Colonization and extinction are major components of the community dynamics at Portal. The metacommunity consists of at least 21 desert rodent species that have geographic ranges overlapping the site. Indeed, all 21 of these species have been caught at the site at least once (Goheen et al. 2005), although only three of these species have been continuously present (fig. 4; Brown et al. 2001). Each of the remaining 18 species colonized or became extinct at least once, with many doing so multiple times (Brown et al. 2001). We are reasonably confident that these colonization and extinction events are not the result of detection issues because (1) sampling intensity on each plot is high,

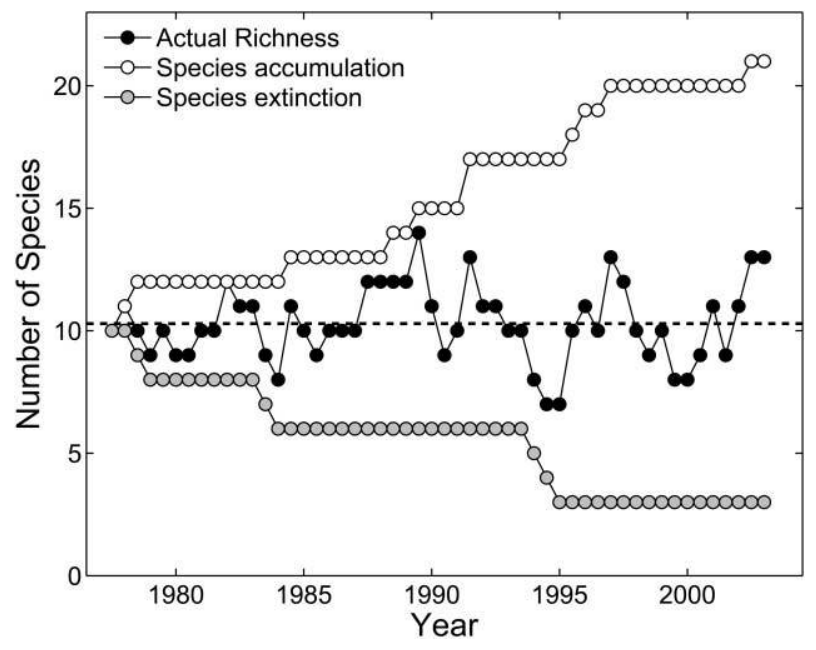

Figure 4: Temporal dynamics (1977-1998) of rodent species richness at Portal, Arizona. Temporal trend in species richness (black circles), cumulative colonizations (i.e., first appearance of a species at the site; white circles), and cumulative extinctions of species that were present at the onset of the study (gray circles). The long-term mean species richness is represented by the dashed line (modified from Brown et al. 2001). 
and each month we typically catch $>80 \%$ of the number of individuals known to be alive (Brown and Zeng 1989), and (2) a species was considered to be extinct only if it was missing for six consecutive months. These approximately complementary colonization and extinction events have maintained an average species richness per 6-month period of 10.3 species. So species diversity and composition were strongly influenced by exchange with the surrounding metacommunity. Without colonization to replace populations that became extinct, diversity would undoubtedly be much lower than observed. It is difficult, however, to estimate how much lower because of the roles of immigration and compensatory dynamics among competing species in maintaining the species in both the local communities and metacommunities.

Closer examination of the colonization and extinction dynamics at the site reveals that they are regulated by both the environmental template and the composition of the rodent community itself. The local climate and habitat serve as important filters, restricting access and affecting local species composition. The dramatic changes in species composition documented during the study suggest that niche characteristics interacted with regional-scale changes in abiotic conditions and vegetation characteristics to increase both extinction rates of grassland-affiliated species and colonization success of shrubland-affiliated species. This compensatory colonization was restricted to the granivorous rodent guild and probably was mediated by the foraging trade-offs discussed in "The Niche." So even though the species were not obvious one-to-one replacements, colonization and extinction events were roughly compensatory, resulting in more constant species richness than would be expected from random turnover events (Goheen et al. 2005, 2006). Such compensatory colonization/extinction dynamics imply that, at any given time, the community is approximately saturated: that is, only a certain number of species can be supported, and colonizations must be matched by an approximately equal number of extinctions (Brown et al. 2001). In addition to habitat filters, local community composition also strongly influenced colonization and extinction rates. As discussed in "The Niche," the presence of kangaroo rats decreased the colonization rates and increased the extinction rates of other granivorous rodents (Valone and Brown 1995). Of the four metacommunity models presented by Leibold et al. (2004), the species-sorting model seems to be the most similar to the scenario we have at Portal, where local composition is determined by colonization interacting with local environmental factors and species interactions. Furthermore, as predicted by a species-sorting model, this interaction between colonization from the metacommunity and local processes has allowed the community to track environmental change and maintain a close associ- ation between the local environment and species composition.

Our results also indicate, however, that the ability of colonization from the metacommunity to maintain a correspondence between composition and the environment is imperfect. This is evidenced by the long time lag (18 years) between the experimental removal of kangaroo rats and the arrival of Chaetodipus baileyi, which was almost certainly due to isolation by distance from suitable habitat. It has been suggested that such lags between availability of a suitable competitor-free environment and colonization by compensatory species are artifacts of the scale of the manipulation (Chase and Leibold 2003). In this case, however, the lag seems largely due to the spatial configuration and other characteristics of the surrounding environment and to the composition of the metacommunity. Historical records show that while C. baileyi was present in the region around Portal, it was neither widespread nor abundant (Findley et al. 1975; Hoffmeister 1986; Ernest and Brown 2001a). The nearest habitat patches known, from museum specimen records, to support C. baileyi before initiation of the kangaroo rat removal in 1977 were $>5 \mathrm{~km}$ from the study site. In a metacommunity context where local extinction events are habitat and species specific, long delays in colonization are not merely possible but probable, depending on the spatial context of the surrounding metacommunity.

Like many studies, research at Portal was designed to focus on community dynamics at a local scale. While local processes are obviously important in governing these dynamics, colonization from surrounding areas is also necessary to account for the observed response to climate change and maintenance of the energetic zero sum. This demonstrated importance of metacommunity dynamics and dispersal limitation, as currently implemented by many neutral models, means that community models that ignore these important regional components will usually be unable to predict the long-term dynamics of communities, especially in response to major perturbations such as climate or habitat change.

\section{Concluding Remarks: What We Do and Do Not (Yet) Understand}

While we have learned much about the ecology of this site in the Chihuahuan Desert, this synthesis of 30 years of research should be viewed more as a progress report than as a definitive treatise. In fact, it raises almost as many questions as it answers. For example, additional theoretical development of a zero-sum, resource-based approach to communities is clearly needed, as is integration with traditional, niche-based approaches (e.g., Lotka-Volterra, $R^{*}$ ), which emphasize niche overlap and population-level in- 
teractions. Is a zero-sum approach an alternative to nichebased models of community dynamics, or does it represent a type of community configuration in which niche overlap in resource use (but not necessarily other axes) is high? Moreover, while our results suggest strongly that granivorous rodents are at an energetic zero sum, we do not have direct measures of seed availability in our system. Empirical studies that explicitly measure limiting resources will be better able to address how resource limitation constrains community assembly, dynamics, and structure.

To date, our work also suggests that behavioral ecology may play an important role in understanding how habitat shifts, distributions of resource availability across microhabitats, and predation risk interact to drive long-term changes in species composition. The "ecology of fear" has long been known to play an important role in microhabitat selection in desert rodents (e.g., Kotler 1984; Brown et al. 1994), and its general importance is gaining wider appreciation (e.g., Ripple and Beschta 2004; Preisser et al. 2005; Creel et al. 2007). While behavioral ecology has not been our focus at Portal, our results do suggest that when habitats shift, the ecology of fear may be critical in driving changes in species' abundances as the landscape of predation risk changes. Clearly, continued work integrating behavioral ecology with community ecology is needed for an understanding of how long-term environmental trends can select for traits and behaviors of species that influence community-level properties.

Finally, our understanding of the role of the metacommunity in the dynamics at our site is obviously insufficient. Where are the refugia for species that disappear and recolonize? We know from the case of Chaetodipus baileyi that time lags can exist between the development of a suitable environment for colonization and the arrival of a new species, but how important are time lags for understanding community dynamics? Is distance from the site more important in delaying colonization than the degree of suitability of the intervening habitat? Finally, what determines the rate of colonization and extinction at the site? Is it controlled more by local dynamics or by regional processes? The study of metacommunity dynamics is a relatively new area of research that has focused mainly on theoretical models (e.g., Wilson 1992; Mouquet and Loreau 2002) and micro- and mesocosm experiments (e.g., Warren 1996; Starzomski and Srivastava 2007), but more work is needed for an understanding of how metacommunity dynamics apply to terrestrial vertebrate communities embedded within environmental gradients.

While there is still much to be understood, there is much we can say with confidence about how dynamics at our site relate to current models of community ecology. Longterm studies provide invaluable information about community assembly and the kinds of models that are nec- essary and sufficient to account for community structure and dynamics (see also Adler 2004; Maurer and McGill 2004). Evidence from Portal reveals the importance of three key processes-energetic zero-sum dynamics, the niche, and metacommunity dynamics - and suggests how they should be included in models of ecological communities. The long-term dynamics of community-level properties such as abundance and aggregate energy use are better explained by a resource-based zero-sum constraint than by a constraint on abundance per se. While the whole community seems to be limited by a near-steady state between rates of resource supply and consumption, individual species responded dramatically to long-term changes in climate and habitat. These shifts in species abundance and community composition were due largely to species-specific niche requirements, especially habitat relations. Other studies on desert rodents suggest that the habitat relations of these species are based not only on patterns of resource availability but also on relative predation risk in different habitat types (Kotler and Brown 1988; Brown et al. 1994). Finally, despite the demonstrated importance of local interactions at the site (Munger and Brown 1981; Heske et al. 1994; Valone and Brown 1995), exchanges of species with the surrounding region were critical in supplying species adapted to new environments, thus allowing the maintenance of both the energetic zero sum and local species richness.

That the interactions among three phenomena-zerosum dynamics, the niche, and metacommunity dynamics-remain poorly understood theoretically and inadequately documented empirically may simply reflect the uniqueness of Portal. Therefore, it would not be surprising if the three phenomena did not play out with precisely the same results on assembly and dynamics at other locales. On the other hand, few studies have the combination of long-term data, experimental manipulations, and background climate and habitat changes that we have had in 30 years at Portal, suggesting that further exploration of these types of long-term dynamics in other systems may provide useful insights into the dynamics and assembly of communities. Certainly, the processes described here and the manner in which they interact with one another may be operating more generally in other systems.

Current models and approaches emphasize different sets of processes that structure communities and therefore are viewed as competing alternatives. It has proven difficult to evaluate support for model predictions because the processes emphasized often are not mutually exclusive or independent. A complementary approach to model development is to evaluate how a suite of mechanisms (e.g., resource competition, niche-based assembly, colonization from the species pool) operate in nature and then implement such processes in future models. Here, we have de- 
rived insights from a well-studied system regarding the role of zero-sum dynamics, the niche, and metacommunity dynamics. The Portal Project provides a novel example of how these factors drive a community's response to environmental changes, ranging from habitat alteration to the removal of dominant species. Ecosystems are expected to undergo both increasing numbers of extinctions and rapid changes in habitat as a result of anthropogenic influences. To address these important ecological issues, the next generation of community models would benefit from the integration of neutral and niche-based processes to better predict long-term dynamics of ecological communities.

\section{Acknowledgments}

We would like to thank the multitudes of volunteers, graduate students, and postdocs who have contributed to the data collection and research at the site. In particular, we would like to thank A. Ernest, who has volunteered almost every month for more than 10 years. The Portal Project is a National Science Foundation (NSF) Long-Term Research in Environmental Biology site, currently supported by the collaborative grants DEB-0702875 and DEB0348255. E.P.W. was supported by an NSF Postdoctoral Fellowship in Biological Informatics.

\section{Literature Cited}

Adler, P. B. 2004. Neutral models fail to reproduce observed speciesarea and species-time relationships in Kansas grasslands. Ecology 85:1265-1272.

Adler, P. B., J. HilleRisLambers, and J. M. Levine. 2007. A niche for neutrality. Ecology Letters 10:95-104.

Bell, G. 2000. The distribution of abundance in neutral communities. American Naturalist 155:606-617.

- 2001. Neutral macroecology. Science 293:2413-2418.

Bowers, M. A., and J. H. Brown. 1982. Body size and coexistence in desert rodents: chance or community structure? Ecology 63:391400.

Bowers, M. A., D. B. Thompson, and J. H. Brown. 1987. Spatialorganization of a desert rodent community: food addition and species removal. Oecologia (Berlin) 72:77-82.

Brady, M. J., and N. A. Slade. 2004. Long-term dynamics of a grassland rodent community. Journal of Mammalogy 85:552-561.

Brown, J. H. 1981. Two decades of homage to Santa Rosalia: toward a general theory of diversity. American Zoologist 21:877-888.

Brown, J. H., and E. J. Heske. 1990. Temporal changes in a Chihuahuan Desert rodent community. Oikos 59:290-302.

Brown, J. H., and J. C. Munger. 1985. Experimental manipulation of a desert rodent community: food addition and species removal. Ecology 66:1545-1563.

Brown, J. H., and Z. Zeng. 1989. Comparative population ecology of eleven species of rodents in the Chihuahuan Desert. Ecology 70:1507-1525.

Brown, J. H., O. J. Reichman, and D. W. Davidson. 1979. Granivory in desert ecosystems. Annual Review of Ecology and Systematics 10:201-227.
Brown, J. H., T. J. Valone, and C. G. Curtin. 1997. Reorganization of an arid ecosystem in response to recent climate change. Proceedings of the National Academy of Sciences of the USA 94:97299733.

Brown, J. H., B. J. Fox, and D. A. Kelt. 2000. Assembly rules: desert rodent communities are structured at scales from local to continental. American Naturalist 156:314-321.

Brown, J. H., S. K. M. Ernest, J. M. Parody, and J. P. Haskell. 2001. Regulation of diversity: maintenance of species richness in changing environments. Oecologia (Berlin) 126:321-332.

Brown, J. H., J. F. Gillooly, A. P. Allen, V. M. Savage, and G. B. West. 2004. Toward a metabolic theory of ecology. Ecology 85:17711789.

Brown, J. S. 1989. Desert rodent community structure: a test of four mechanisms of coexistence. Ecological Monographs 59:1-20.

Brown, J. S., B. P. Kotler, R. J. Smith, and W. O. Wirtz. 1988. The effects of owl predation on the foraging behavior of heteromyid rodents. Oecologia (Berlin) 76:408-415.

Brown, J. S., B. P. Kotler, and W. A. Mitchell. 1994. Foraging theory, patch use, and the structure of a Negev Desert granivore community. Ecology 75:2286-2300.

Calder, W. A. 1984. Size, function, and life history. Dover, Mineola, NY.

Caswell, H. 1976. Community structure: a neutral model analysis. Ecological Monographs 46:327-354.

Chase, J. M., and M. A. Leibold. 2003. Ecological niches. University of Chicago Press, Chicago.

Chase, J. M., W. G. Wilson, and S. A. Richards. 2001. Foraging tradeoffs and resource patchiness: theory and experiments with a freshwater snail community. Ecology Letters 4:304-312.

Chave, J. 2004. Neutral theory and community ecology. Ecology Letters 7:241-253.

Chave, J., H. C. Muller-Landau, and S. A. Levin. 2002. Comparing classical models: theoretical consequences for patterns of diversity. American Naturalist 159:1-23.

Chave, J., D. Alonso, and R. S. Etienne. 2006. Comparing models of species abundance. Nature 441:E1.

Chesson, P. 2000. Mechanisms of maintenance of species diversity. Annual Review of Ecology and Systematics 31:343-366.

Clark, J. S., and J. S. McLachlan. 2003. Stability of forest biodiversity. Nature 423:635-638.

Creel, S., D. Christianson, S. Liley, and J. A. Winnie. 2007. Predation risk affects reproductive physiology and demography of elk. Science 315:960.

Damuth, J. 1981. Population density and body size in mammals. Nature 290:699-700.

Davidson, D. W., R. S. Inouye, and J. H. Brown. 1984. Granivory in a desert ecosystem: experimental evidence for indirect facilitation of ants by rodents. Ecology 65:1780-1786.

del Monte-Luna, P., B. W. Brook, M. J. Zetina-Rejon, and V. H. CruzEscalona. 2004. The carrying capacity of ecosystems. Global Ecology and Biogeography 13:485-495.

Enquist, B. J., J. Sanderson, and M. D. Weiser. 2002. Modeling macroscopic patterns in ecology. Science 295:1835-1836.

Ernest, S. K. M. 2005. Body size, energy use, and community structure of small mammals. Ecology 86:1407-1413.

Ernest, S. K. M., and J. H. Brown. 2001a. Delayed compensation for missing keystone species by colonization. Science 292:101-104.

. $2001 \mathrm{~b}$. Homeostasis and compensation: the role of species and resources in ecosystem stability. Ecology 82:2118-2132. 
Ernest, S. K. M., J. H. Brown, and R. R. Parmenter. 2000. Rodents, plants, and precipitation: spatial and temporal dynamics of consumers and resources. Oikos 88:470-482.

Etienne, R. S., D. Alonso, and A. J. McKane. 2007. The zero-sum assumption in neutral biodiversity theory. Journal of Theoretical Biology 248:522-536.

Findley, J. S., A. H. Harris, D. E. Wilson, and C. Jones. 1975. Mammals of New Mexico. University of New Mexico Press, Albuquerque.

Frost, T. M., S. R. Carpenter, A. R. Ives, and T. K. Kratz. 1995. Species compensation and complementarity in ecosystem function. Pages 224-239 in C. G. Jones and J. H. Lawton, eds. Linking species and ecosystems. Chapman \& Hall, New York.

Genoways, H. H., and J. H. Brown, eds. 1993. Biology of the family Heteromyidae. Special Publication 10. American Society of Mammalogists, Shippensburg, PA.

Goheen, J. R., E. P. White, S. K. M. Ernest, and J. H. Brown. 2005. Intra-guild compensation regulates species richness in desert rodents. Ecology 86:567-573.

- 2006. Intra-guild compensation regulates species richness in desert rodents: reply. Ecology 87:2121-2125.

Gravel, D., C. D. Canham, M. Beaudet, and C. Messier. 2006. Reconciling niche and neutrality: the continuum hypothesis. Ecology Letters 9:399-409.

Heske, E. J., J. H. Brown, and S. Mistry. 1994. Long-term experimental study of a Chihuahuan Desert rodent community: 13 years of competition. Ecology 75:438-445.

Hoffmeister, D. F. 1986. Mammals of Arizona. University of Arizona Press, Tucson.

Holmgren, M., P. Stapp, C. R. Dickman, C. Gracia, S. Graham, J. R. Gutiérrez, C. Hice, et al. 2006. Extreme climatic events shape arid and semiarid ecosystems. Frontiers in Ecology and the Environment 4:87-95.

Holyoak, M., and M. Loreau. 2006. Reconciling empirical ecology with neutral community models. Ecology 87:1370-1377.

Holyoak, M., M. A. Leibold, N. M. Mouquet, R. D. Holt, and M. F. Hoopes. 2005. Metacommunities: a framework for large-scale community ecology. Pages 1-31 in M. Holyoak, M. A. Leibold, and R. D. Holt, eds. Metacommunities: spatial dynamics and ecological communities. University of Chicago Press, Chicago.

Houlahan, J. E., D. J. Currie, K. Cottenie, G. S. Cummings, S. K. M. Ernest, C. S. Findlay, S. D. Fuhlendorf, et al. 2007. Compensatory dynamics are rare in natural ecological communities. Proceedings of the National Academy of Sciences of the USA 104: 3273-3277.

Hubbell, S. P. 1979. Tree dispersion, abundance, and diversity in a tropical dry forest. Science 203:1299-1309.

. 2001. The unified neutral theory of biodiversity and biogeography. Princeton University Press, Princeton, NJ.

- 2005. Neutral theory in community ecology and the hypothesis of functional equivalence. Functional Ecology 19:166-172.

- 2006. Neutral theory and the evolution of ecological equivalence. Ecology 87:1387-1398.

Hughes, R. G. 1984. A model of the structure and dynamics of benthic marine invertebrate communities. Marine Ecology Progress Series 15:1-11.

Karlson, R. H., and H. V. Cornell. 2002. Species richness of coral assemblages: detecting regional influences at local spatial scales. Ecology 83:452-463.
Keitt, T. H., and J. Fischer. 2006. Detection of scale-specific community dynamics using wavelets. Ecology 87:2895-2904.

Kelt, D. A., P. L. Meserve, M. L. Forister, L. K. Nabors, and J. R. Gutiérrez. 2004. Seed predation by birds and small mammals in semiarid Chile. Oikos 104:133-141.

Kleiber, M. 1932. Body size and metabolism. Hilgardia 6:315-332.

Kneitel, J. M., and J. M. Chase. 2004. Trade-offs in community ecology: linking spatial scales and species coexistence. Ecology Letters 7:69-80.

Kotler, B. P. 1984. Risk of predation and the structure of desert rodent communities. Ecology 65:689-701.

Kotler, B. P., and J. S. Brown. 1988. Environmental heterogeneity and the coexistence of desert rodents. Annual Review of Ecology and Systematics 19:281-307.

Kotler, B. P., J. S. Brown, and W. A. Mitchell. 1994. The role of predation in shaping the behavior, morphology, and community organization of desert rodents. Australian Journal of Zoology 42: 449-466.

Lawton, J. H. 1999. Are there general laws in ecology? Oikos 84:177192.

Leibold, M. A., and M. A. McPeek. 2006. Coexistence of the niche and neutral perspectives in community ecology. Ecology 87:13991410.

Leibold, M. A., M. Holyoak, N. Mouquet, P. Amarasekare, J. M. Chase, M. F. Hoopes, R. D. Holt, et al. 2004. The metacommunity concept: a framework for multi-scale community ecology. Ecology Letters 7:601-613.

Levinton, J. S. 1979. A theory of diversity equilibrium and morphological evolution. Science 204:335-336.

MacArthur, R. H. 1957. On the relative abundance of bird species. Proceedings of the National Academy of Sciences of the USA 43: 293-295.

MacArthur, R. H., and R. Levins. 1967. The limiting similarity, convergence, and divergence of coexisting species. American Naturalist 101:377-385.

MacArthur, R. H., and E. O. Wilson. 1967. The theory of island biogeography. Princeton University Press, Princeton, NJ.

Maurer, B. A., and B. J. McGill. 2004. Non-neutral ecology. Basic and Applied Ecology 5:413-422.

McGill, B. J. 2003a. Strong and weak tests of macroecological theory. Oikos 102:679-685.

- 2003b. A test of the unified neutral theory of biodiversity. Nature 422:881-885.

McGill, B. J., E. A. Hadly, and B. A. Maurer. 2005. Community inertia of Quaternary small mammal assemblages in North America. Proceedings of the National Academy of Sciences of the USA 102: 16701-16706.

Meserve, P. L., W. B. Milstead, and J. R. Gutiérrez. 2001. Results of a food addition experiment in a north-central Chile small mammal assemblage: evidence for the role of "bottom-up" factors. Oikos 94:548-556.

Morgan, K. R., and M. V. Price. 1992. Foraging in heteromyid rodents: the energy cost of scratch-digging. Ecology 73:2260-2272.

Mouquet, N., and M. Loreau. 2002. Coexistence in metacommunities: the regional similarity hypothesis. American Naturalist 159: 420-426.

- 2003. Community patterns in source-sink metacommunities. American Naturalist 162:544-557.

Munger, J. C., and J. H. Brown. 1981. Competition in desert rodents: 
an experiment with semipermeable exclosures. Science 211:510512.

Neilson, R. P. 1986. High-resolution climatic analysis and southwest biogeography. Science 232:27-34.

Nekola, J. C., and J. H. Brown. 2007. The wealth of species: ecological communities, complex systems, and the legacy of Frank Preston. Ecology Letters 10:188-196.

Ostfeld, R. S., and F. Keesing. 2000. Pulsed resources and community dynamics of consumers in terrestrial ecosystems. Trends in Ecology \& Evolution 15:232-237.

Peters, R. H. 1983. The ecological implications of body size. Cambridge University Press, Cambridge.

Preisser, E. L., D. I. Bolnick, and M. F. Benard. 2005. Scared to death? the effects of intimidation and consumption in predator-prey interactions. Ecology 86:501-509.

Price, M. V. 1978. The role of microhabitat in structuring desert rodent communities. Ecology 59:910-921.

- 1983. Ecological consequences of body size: a model for patch choice in desert rodents. Oecologia (Berlin) 59:384-392.

. 1993. A functional-morphometric analysis of forelimbs in bipedal and quadrupedal heteromyid rodents. Biological Journal of the Linnean Society 50:339-360.

Price, M. V., and J. E. Mittler. 2003. Seed-cache exchange promotes coexistence and coupled consumer oscillations: a model of desert rodents as resource processors. Journal of Theoretical Biology 223: 215-231.

. 2006. Cachers, scavengers, and thieves: a novel mechanism for desert rodent coexistence. American Naturalist 168:194-206.

Reichman, O. J., and D. Oberstein. 1977. Selection of seed distribution types by Dipodomys merriami and Perognathus amplus. Ecology 58:636-643.

Reichman, O. J., and M. V. Price. 1993. Ecological aspects of heteromyid foraging. Pages 539-574 in $\mathrm{H}$. H. Genoways and J. H. Brown, eds. Biology of the family Heteromyidae. Special Publication 10. American Society of Mammalogists, Shippensburg, PA.

Richards, S. A., R. M. Nisbet, W. G. Wilson, and H. P. Possingham. 2000. Grazers and diggers: exploitation competition and coexistence among foragers with different feeding strategies on a single resource. American Naturalist 155:266-279.

Ripple, W. J., and R. L. Beschta. 2004. Wolves and the ecology of fear: can predation risk structure ecosystems? BioScience 54:755766.

Root, R. B. 1973. Organization of a plant-arthropod association in simple and diverse habitats: the fauna of collards (Brassica oeracea). Ecological Monographs 43:95-124.

Ruhl, H. A., and K. L. Smith Jr. 2004. Shifts in deep-sea community structure linked to climate and food supply. Science 305:513-515.

Schoener, T. W. 1974. Resource partitioning in ecological communities. Science 185:27-39.

Simberloff, D. 2004. Community ecology: is it time to move on? American Naturalist 163:787-799.

Smith, F. A., J. H. Brown, and T. J. Valone. 1997. Path analysis: a critical evaluation using long-term experimental data. American Naturalist 149:29-42.

Snyder, R. E., and P. Chesson. 2004. How the spatial scales of dispersal, competition, and environmental heterogeneity interact to affect coexistence. American Naturalist 164:633-650.

Starzomski, B. M., and D. S. Srivastava. 2007. Landscape geometry determines community response to disturbance. Oikos 116:690699.
Stevens, R. D., C. López-González, and S. J. Presley. 2007. Geographical ecology of Paraguayan bats: spatial integration and metacommunity structure of interacting assemblages. Journal of Animal Ecology 76:1086-1093.

Thibault, K. M., and J. H. Brown. 2008. Impact of an extreme climatic event on community assembly. Proceedings of the National Academy of Sciences of the USA 105:3410-3415.

Thibault, K. M., E. P. White, and S. K. M. Ernest. 2004. Temporal dynamics in the structure and composition of a desert rodent community. Ecology 85:2649-2655.

Thompson, D. B., J. H. Brown, and W. D. Spencer. 1991. Indirect facilitation of granivorous birds by desert rodents: experimental evidence from foraging patterns. Ecology 72:852-863.

Tilman, D. 1982. Resource competition and community structure. Princeton University Press, Princeton, NJ.

- 2004. Niche tradeoffs, neutrality, and community structure: a stochastic theory of resource competition, invasion, and community assembly. Proceedings of the National Academy of Sciences of the USA 101:10854-10861.

Underwood, A. J., and P. G. Fairweather. 1989. Supply-side ecology and benthic marine assemblages. Trends in Ecology \& Evolution 4:16-20.

Valone, T. J., and N. A. Barber. 2008. An empirical evaluation of the insurance hypothesis in diversity-stability models. Ecology 89:522531.

Valone, T. J., and J. H. Brown. 1995. Effects of competition, colonization, and extinction on rodent species diversity. Science 267: $880-883$.

-1996. Desert rodents: long-term responses to natural changes and experimental manipulation. Pages 555-584 in M. L. Cody and J. A. Smallwood, eds. Long-term studies of vertebrate communities. Academic Press, San Diego, CA.

Valone, T. J., J. H. Brown, and C. L. Jacobi. 1995. Catastrophic decline of a desert rodent, Dipodomys spectabilis: insights from a longterm study. Journal of Mammalogy 76:428-436.

Van Valen, L. 1973. A new evolutionary law. Evolutionary Theory 1: $1-30$.

. 1976. Energy and evolution. Evolutionary Theory 1:179229.

Vasseur, D. A., and U. Gaedke. 2007. Spectral analysis unmasks synchronous and compensatory dynamics in plankton communities. Ecology 88:2058-2071.

Vasseur, D. A., U. Gaedke, and K. S. McCann. 2005. A seasonal alternation of coherent and compensatory dynamics occurs in phytoplankton. Oikos 110:507-514.

Volkov, I., J. R. Banavar, S. P. Hubbell, and A. Maritan. 2003. Neutral theory and relative species abundance in ecology. Nature 424:10351037.

Volkov, I., J. R. Banavar, F. L. He, S. P. Hubbell, and A. Maritan. 2005. Density dependence explains tree species abundance and diversity in tropical forests. Nature 438:658-661.

2006. Comparing models of species abundance: reply. Nature 441:E1-E2.

Warren, P. H. 1996. Dispersal and destruction in a multiple habitat system: an experimental approach using protist communities. Oikos 77:317-325.

Werner, E. E., K. L. Yurewicz, D. K. Skelly, and R. A. Relyea. 2007. Turnover in an amphibian metacommunity: the role of local and regional factors. Oikos 116:1713-1725.

West, G. B., J. H. Brown, and B. J. Enquist. 1997. A general model 
for the origins of allometric scaling laws in biology. Science 276: $122-126$.

White, E. P., S. K. M. Ernest, and K. M. Thibault. 2004. Trade-offs in community properties through time in a desert rodent community. American Naturalist 164:670-676.

White, E. P., S. K. M. Ernest, A. J. Kerkhoff, and B. J. Enquist. 2007. Relationships between body size and abundance in ecology. Trends in Ecology \& Evolution 22:323-330.

Wilson, D. S. 1992. Complex interactions in metacommunities, with implications for biodiversity and higher levels of selection. Ecology 73:1984-2000.

Wootton, J. T. 2005. Field parameterization and experimental test of the neutral theory of biodiversity. Nature 433:309-312.

Wright, D. H. 1983. Species-energy theory: an extension of speciesarea theory. Oikos 41:496-506.

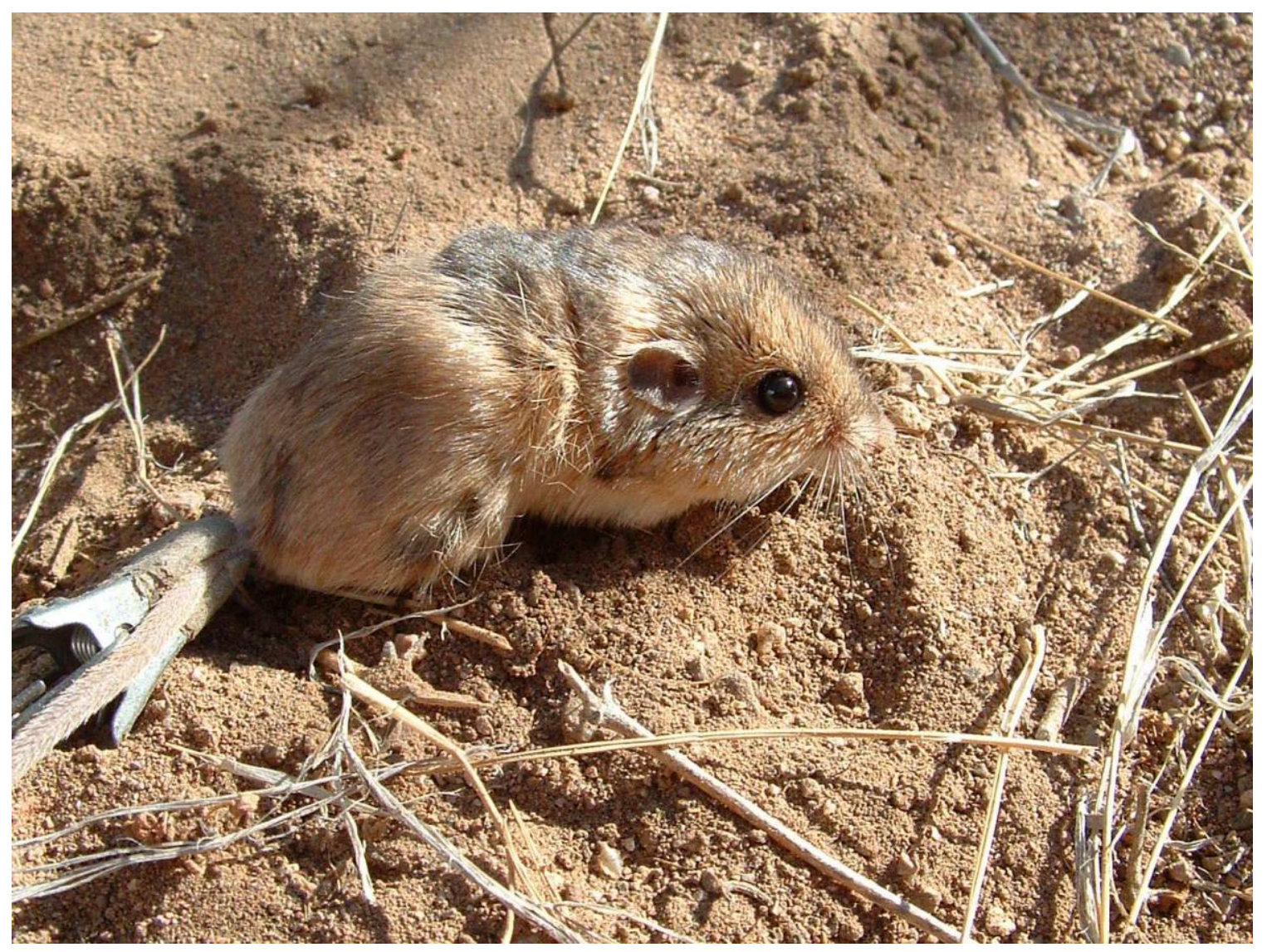

The silky pocket mouse (Perognathus flavus) is one of the seed-eating desert rodents studied at the long-term site at Portal, Arizona (photograph by Travis Perry). 